\title{
Hepatic Tumor Detection using U-Net++ from Lungs CT Images
}

\author{
Md Haris Uddin Sharif* \\ * Department of Information Technology, University of the Cumberlands \\ DOI: 10.29322/IJSRP.11.11.2021.p11903 \\ http://dx.doi.org/10.29322/IJSRP.11.11.2021.p11903
}

\begin{abstract}
Cancer is today's one of the most harmful and deadly diseases. Early detection of cancer is essential as it helps radiologists and doctors perform treatment planning and surgery. Detection of tumors in the early stages is a difficult task. Artificial intelligencebased approaches which use deep learning and machine learning techniques assist radiologists in the prediction. The main aim of this research is to develop a fully automated tool for Liver Tumor Segmentation. Also, the purpose of this research is to examine the problem of lungs cancer detection from CT lungs images and propose an efficient approach that will segment out complex regions and improves the performance with lesser error rates. These techniques not only detect tumor but also provides other information (Survival rate, tumor statistics) which are very helpful.
\end{abstract}

Index Terms- Deep Learning, Custom Design CNN, Data distribution, Loss Function, Evaluation Metrics, Optimizer, Machine Learning, Liver Tumor Segmentation, CT Scans, 3D-IRCADb Dataset.

\section{INTRODUCTION}

A large percentage (\%) of today's population can be affected by cancer throughout its lifetime. The earlier cancer is identified, the better the life likelihood. Proper radiological detection can obtain the difference between life and death. Primary liver tumor, known as hepatocellular carcinoma (HCC), and secondary liver tumor (metastasis) represent, in aggregate, the second most common cause of cancer-related mortality in the World. Existing Computed Assisted Diagnosis (CADs) software uses traditional image analysis techniques. Their applications are mainly oriented towards the type of cancer that shows specific imaging characteristics (e.g., lung nodules, colon polyps, and breast micro calcifications). This project aims to innovate in the field of automatic medical image analysis by using the latest developments in artificial intelligence, computer vision, and machine learning. Using readily available data of sufficient quantity from the liver through extensive datasets hand-segmented, we plan to develop a framework that will detect and segment tumors/lesions in a liver segmented from CT images. This approach opens new prospects for medical imaging applications by proposing models that exhibit high accuracy with minimal human intervention, broadening their use and ultimately improving patient care.

\section{IDENTIFY, RESEARCH AND COLLECT IDEA}

Accurate diagnosis can vary between life and death as early diagnoses lead to a better prognosis. In terms of cancer-related mortality, primary liver tumors such as hepatocellular carcinoma (HCC) and secondary liver tumors (metastasis) are second only to lung cancer in the world. Traditional Computer Aided Diagnostics (CAD) systems use machine learning and image processing techniques. They have less performance and focused on the type of malignancy belonging to imaging characteristics (e.g., MRI scans of the brain, breast, etc.) Existing Computed Assisted Diagnosis (CADs) software uses traditional image analysis techniques. Their applications are mainly oriented towards the type of cancer that shows specific imaging characteristics (e.g., lung nodules, colon polyps, and breast microcalcifications). With the advent of deep learning, the performance of these systems improves from 5 to $10 \%$ and shown robust performance and effective results. This project aims to build an end-to-end architecture for the segmentation of tumors in lungs images. We intend to design a framework that will detect and segment liver tumors/lesions in CT images using readily available liver data from massive datasets that have been segmented. Using models that display great accuracy with low human intervention, this strategy expands the usage of medical imaging applications and, as a result, leads to better patient care for everyone involved. This project aims to innovate in the field of automatic medical image analysis by using the latest developments in artificial intelligence, computer vision, and machine learning. Using readily available data of sufficient quantity from the liver through extensive datasets hand-segmented, we plan to develop a framework that will detect and segment tumors/lesions in a liver segmented from CT images. 
This approach opens new prospects for medical imaging applications by proposing models that exhibit high accuracy with minimal human intervention, broadening their use and ultimately improving patient care.

\section{LITERATURE REVIEW}

Several studies have been conducted for the detection of tumors using lung scans. These techniques are categorized into machine learning and deep learning techniques. Different image processing techniques, including region, growing, watershed segmentation, template matching, edges detection algorithms, and fusion of additional image processing techniques, are proposed to segment various lung diseases that cover tumors detection [1]. Some approaches perform segmentation or detection using image processing techniques. In contrast, others use image processing-based methods for features extraction, and then machine learning algorithms (SVM, Decision Tree, and KNN) are trained on the selected relevant features. Feature selection techniques are also incorporated for relevant features selection. Due to imaging machine settings, the scans are not uniformly the same. For noise removal and registration, image processing approaches are also used.

Deep learning approaches are widely adopted for this problem as the end-to-end method automatically extracts features, and the relevant components are selected using the activation function approaches. A CAD-based system that uses Convolutional Neural Network (CNN) based architecture for segmentation of lung cancer is proposed in [2]. Their proposed approach achieves good results and performs segmentation in a short duration. Similarly, a Deep Belief Networks (DBN) and Stack Denoising Autoencoder (SNAE) combined technique is proposed in [3]. An automatic end-to-end deep learning-based approach is proposed in [4], in which the author proposed a CNN-based model in which they first normalize the images to keep their values in some range, and then the photos are trained on the deep learning model. Generative models are also proposed for lung cancer detection. Instead of discriminative models, they use probability distribution to find the underlying data distribution to generate similar images or classify them. Generative models are based on the game theory concept in which multiple players try to win and reach the Pareto optimal state. In [4], authors proposed CT-GAN (Conditional Term- Generative Adversarial Networks) for malicious tampering of 3D images.

Their approach is to detect malicious or tempered images to protect the original data. Similarly, in [5], a GAN-based augmentation approach is combined with a deep SEED model for detection. They have used CT (Computer Tomography) scans images for experiments. The experimental results show an improvement of 3\% sensitivity after combining the augmented data with the training data. They achieved $91 \%$ sensitivity and $86 \%$ specificity. Annotation of data is no doubt one of the major tasks faced by researchers and developers. Data annotation is a time-consuming and costly task. To overcome this solution, semi-supervised or unsupervised based methods for lungs cancer detection are proposed in the literature. These methods use limited training data and constrictive learning-based techniques to estimate the probability distribution or the data to learn the new patterns and fit on the data. In [6], weekly supervised-based lungs detection first used patch-based deep CNN architecture to select a discriminative patch that contains essential information. Then different context-aware blocks are combined with feature aggregation blocks to capture data, and then the selected data is fed into Random Forest (RF) classifier for prediction. Their proposed approach achieves good results with limited training data. They have combined machine learning and deep learning techniques.

In most of the tasks, encoder-decoder-based architecture was proposed for the said problem. The idea behind encoder-decoder is that the encoder compressed the information into a single vector containing only relevant data. Then the decoder reconstructs the knowledge to build the segmented image. One of the famous architectures is U-Net [7].

Different variants of U-Net were proposed in the literature in which U-Net++ [8]. A typical U-Net++ architecture starts with an encoder sub-network or backbone followed by a decoder sub-network. There are re-designed skip pathways (green and blue) connecting the two sub-networks and deep supervision (red). The architecture of U-Net++ is shown in the below diagram. 


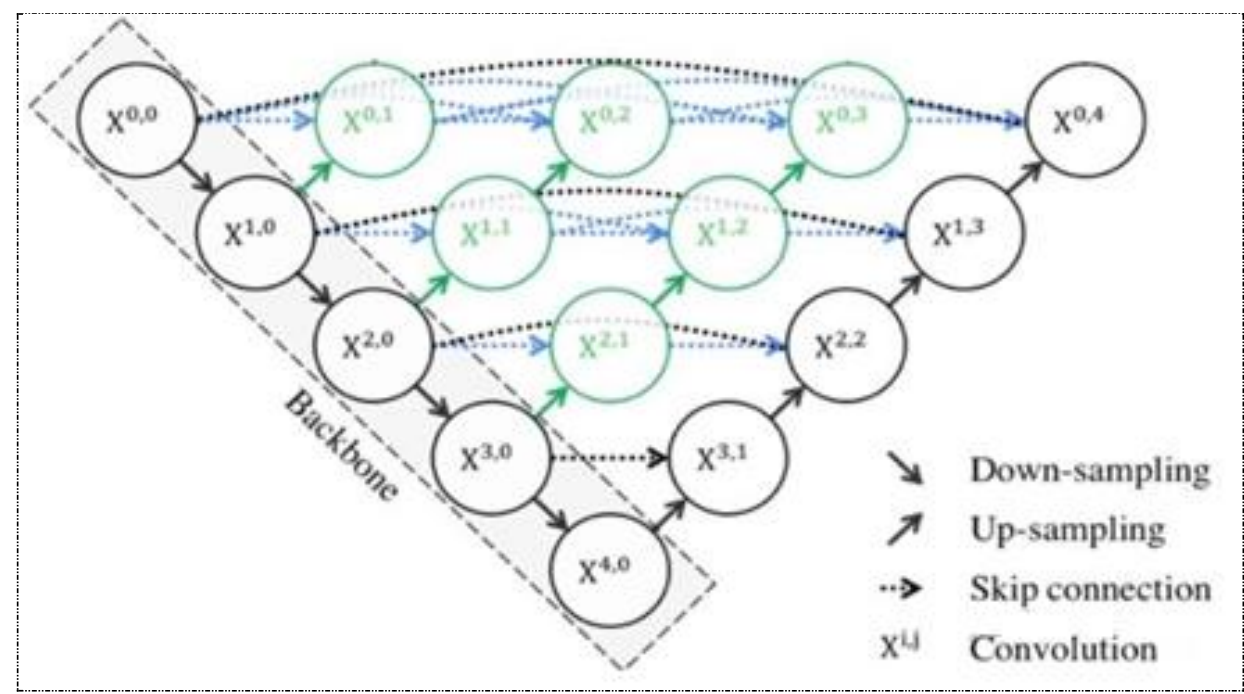

Motivated from this, we have proposed an approach to hepatic segmentation tumors from lung CT images. We have used an opensource dataset for experiments and use dice loss function, which is based on dice coefficient.

\section{EXPERIMENTAL SETTINGS}

A batch size of 8 is used to perform the experiments, and 100 epochs and an initial learning rate of 0.0001 are set. The dice loss function is used. We have used Google Colab with a GPU of $12 \mathrm{~GB}$, Ram of $12 \mathrm{~GB}$, and Pytorch as a framework for experiments. The experiments took about 4 hours to train the model. Adam optimizer is used as an optimization technique with early stopping technique for premature convergence of model.

\section{LOSS FUNCTION}

The dice loss function is based on the dice coefficient, which computes the similarity between two images. It was developed in 1940 to find similarities between two samples. It was proposed for 3D medical image segmentation tasks by Milletari et al., widely used in different computer vision-related tasks [8].

$$
D=\frac{2 \sum_{i=1}^{N} p_{i} g_{i}}{\sum_{i=1}^{N} p_{i}{ }^{2}+\sum_{i=1}^{N} g_{i}{ }^{2}}
$$

Equation (1) shows the dice coefficient formula with ground truth image $g$ and predicted image as $p$. The pixel values of predicted and actual images are represented as pi and gi. These pixels values are represented as 0 or 1 . In the case of detection, 1 represents the pixel is in the boundary and 0 as not in the edge. The equation denominator represents the sum of actual and predicted values, and the numerator is the sum of actual predicted values. 


\section{STEPS FOR RUNNING SOURCE CODE}

The code simply run on the GOOGLE COLAB platform we don't need to install any library. Source editor is availablehttps://colab.research.google.com/\#create=true

If we are using on our own Machine with GPU install pytorch version in specific environment conda create -n env python=3.6 conda install pytorch $==0.4 .0$ torchvision $==0.2 .1-\mathrm{c}$ pytorch

Unzip the Dataset and copy the path to the code in the following line. DATASET_PATH = os.path.join('/content/original')

\section{PROPOSED APPROACH}

The proposed approach for segmentation of tumor in lungs image is shown visually in Figure 2. The whole methodology consists of 6 steps in which first, the images are resized into the 512x512 size and converted into the grayscale. In the second stage, the dataset is splatted into 70/30, where $70 \%$ represent the training images and $30 \%$ represent the testing images. After splitting the dataset, the training images are trained into U-Net++ architecture and fine-tuned. After fine-tuning, the trained model is evaluated into the testing set.

\section{U-NET++ ARCHITECTURE}

For training, we have used the U-Net++ architecture. The U-Net approach has numerous advantages for segmentation tasks: first, it allows for the simultaneous use of global location and context. Second, it is effective with a small number of training samples and improved performance for tasks involving segmentation.

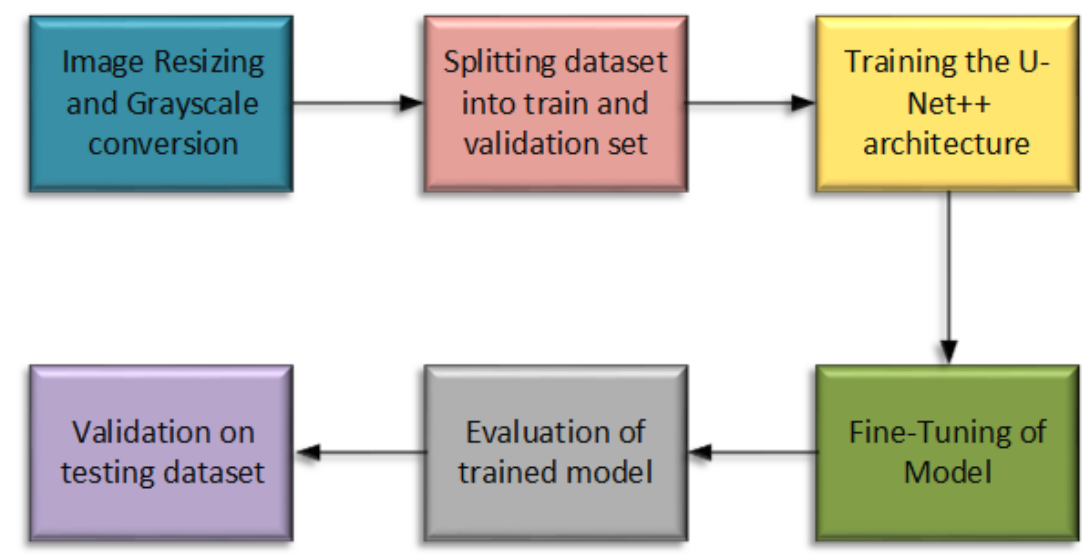

Figure 1 System Model of whole methodology

\section{Results}

The experimental results on training, validation and test set dice score are shown in Table 1. On the training set, the proposed approach DSC score of 0.87 is achieved, 081 is achieved using validation set and the test set evaluation shows the 0.78 DSC score.

Table 1 shows the Validation and Test Dice score of Liver Tumor Segmentation Task.

\begin{tabular}{|c|c|c|c|c|}
\hline Tasks & & Training Score & Validation Score & Test Score \\
\hline $\begin{array}{l}\text { Liver } \\
\text { Segmentation }\end{array}$ & Tumor & 0.87341 & 0.81665 & 0.7884 \\
\hline
\end{tabular}

This publication is licensed under Creative Commons Attribution CC BY. 
For the visual assessment of results, we also computed the predicted mask different with actual to find the pixel values missed during the assessment of model on testing set. The computed differences with actual and predicted masks along with CT scans are shown in Figure 3.

\section{Name: 67.png Dice Score: 0.85015}
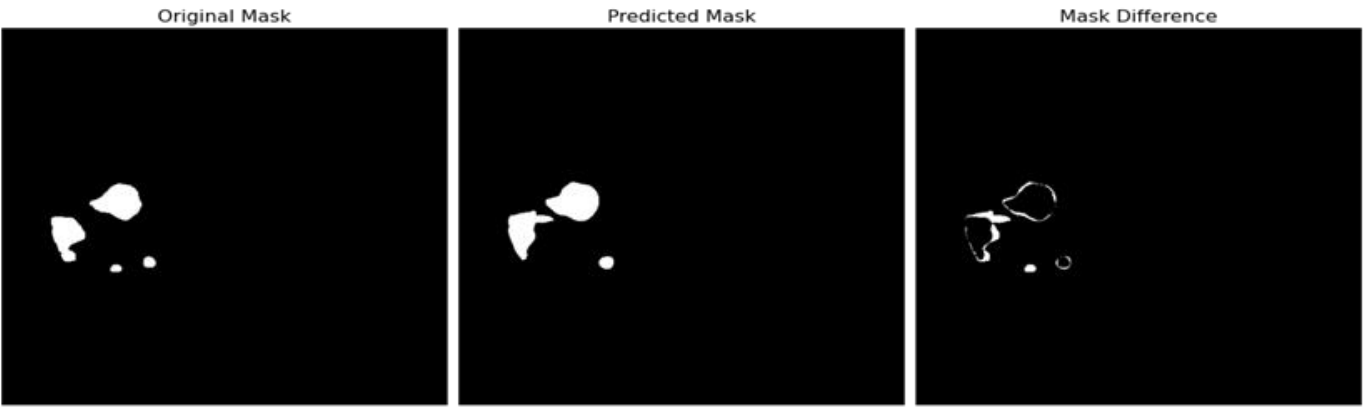

Original Segmentation

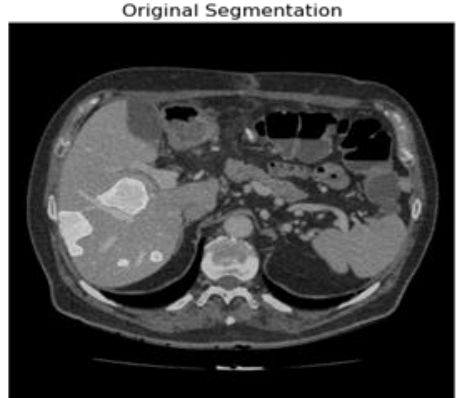

Predicted Segmentation
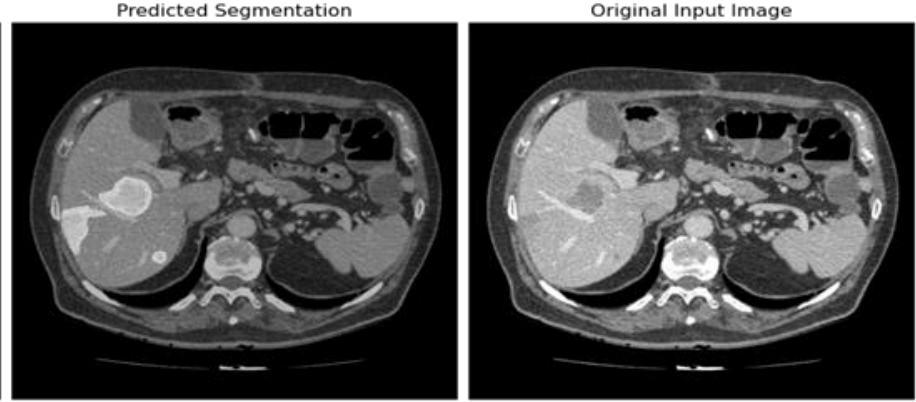

Name: 151.png Dice Score: 0.95554

Original Mask

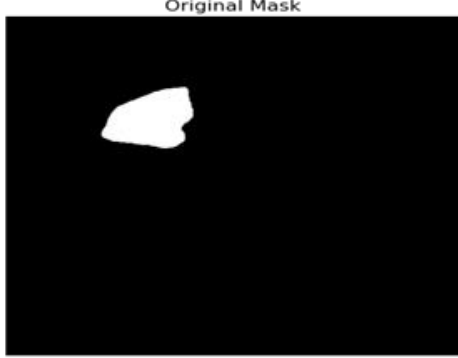

Original Segmentation

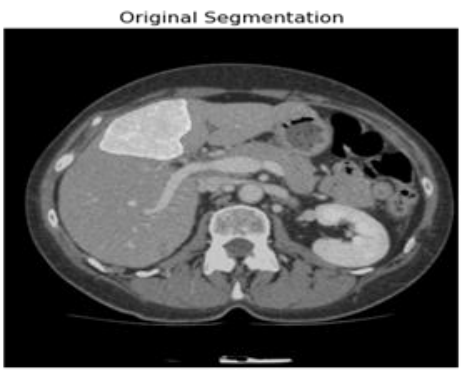

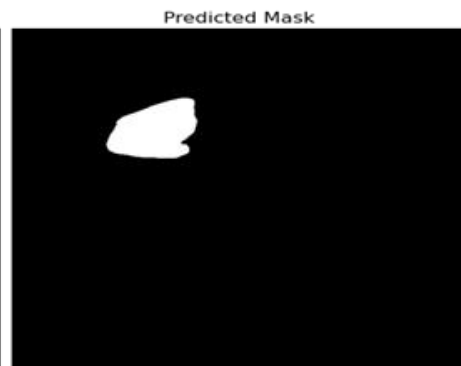

Predicted Segmentation

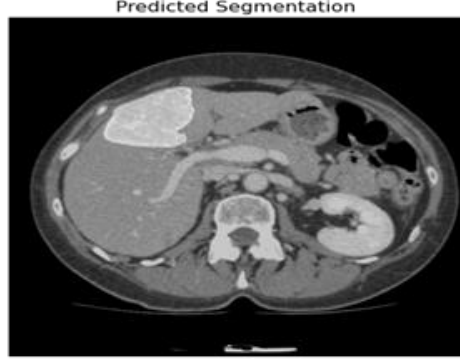

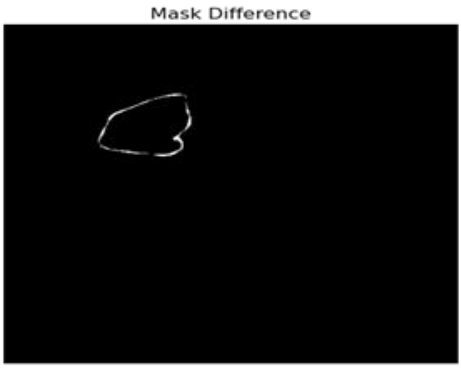

Original Input Image

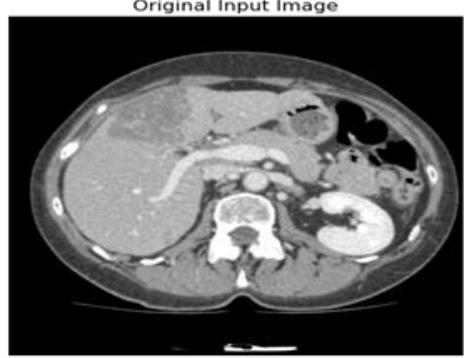

These images are from two patients who show that the predicted mask is nearly similar to the original cover and the difference region shows only the limited pixel area. Furthermore, the proposed approach also segments the complex part, making it quite good for the said approach.

\section{Dataset Details}

For experiments, we have used 3D-IRCADb (3D Image Reconstruction for Comparison of Algorithm Database), which includes multiple organs images of patients. Expert radiologists build the ground truth of this database. These images consist of hepatic tumors of 10 women and ten men, with $75 \%$ of cases having tumors [9]. They have both 3D and 2D slices. The 3D pieces are captured with $1.00 \mathrm{~mm}$ depth and registered and normalized concerning the image details. 


\section{CONCLUSION}

In this study, we have proposed an end-to-end approach for the segmentation of lungs tumor from scans. The proposed approach is validated on testing and validation set, which is unseen data. The evaluation of the proposed system visually and descriptive shows the effectiveness of the proposed method. The future work includes using different data augmentation techniques to increase the data. Furthermore, class inequality techniques should also be incorporated to increase the sensitivity rate. Moreover, semi-supervised and unsupervised learning should also be utilized, which will improve the performance with fewer data.

\section{REFERENCES}

[1] Mutiullah, A., et al. "Lungs cancer detection using digital image processing techniques: a review." Mehran University Research Journal of Engineering and Technology 38.2 (2019): 351-360.

[2] Bhatia, Siddharth, Yash Sinha, and Lavika Goel. "Lung cancer detection: a deep learning approach." Soft Computing for Problem Solving. Springer, Singapore, 2019. 699-705.

[3] Hua, K.-L., et al.: Computer-aided classification of lung nodules on computed tomography images via deep learning technique. Onco Targets Therapy 8 2015$2022(2014)$

[4] Mirsky, Yisroel, et al. "CT-GAN: Malicious tampering of 3D medical imagery using deep learning." 28th \{USENIX\} Security Symposium (\{USENIX\} Security 19). 2019.

[5] Kryuchkov, Maksim, et al. "CT images GAN-based augmentation with AdaIN for lung nodules detection." Thirteenth International Conference on Machine Vision. Vol. 11605. International Society for Optics and Photonics, 2021.

[6] Wang, Xi, et al. "Weakly supervised deep learning for whole slide lung cancer image analysis." IEEE transactions on cybernetics 50.9 (2019): $3950-3962$.

[7] Ronneberger, Olaf, Philipp Fischer, and Thomas Brox. "U-net: Convolutional networks for biomedical image segmentation." International Conference on Medical image computing and computer-assisted intervention. Springer, Cham, 2015.

[8] Milletari, Fausto, Nassir Navab, and Seyed-Ahmad Ahmadi. "V-net: Fully convolutional neural networks for volumetric medical image segmentation." 2016 fourth international conference on 3D vision (3DV). IEEE, 2016.

[9] Legales, M. COVID19: IRCAD sanitary measures \& health pass.https://www.ircad.fr/research/3d-ircadb-02/.2021.

\section{AUTHORS}

Md Haris Uddin Sharif (Graduate Student Member, IEEE) is currently pursuing the Ph.D. degree in information technology with the University of the Cumberlands, Williamsburg, KY, USA. 